\title{
Ionic and nonspherical polymer nanoparticles in nonpolar solvents
}

Gregory N. Smith, ${ }^{*, \dagger}$ Sarah L. Canning, ${ }^{\dagger,}$ Matthew J. Derry, ${ }^{\dagger, \S}$ Elizabeth R. Jones, ${ }^{\dagger}, \|$ Thomas J. Neal, ${ }^{\dagger}$ and Andrew J. Smith ${ }^{\perp}$

$\dagger$ Department of Chemistry, University of Sheffield, Brook Hill, Sheffield, South Yorkshire, S3 7HF, United Kingdom

$\ddagger$ Current address: Niels Bohr Institute, University of Copenhagen, H. C. Ørsted Institute, Universitetsparken 5, 2100 Copenhagen Ø, Denmark

ICurrent address: Fujifilm Speciality Ink Systems Ltd, Pysons Road, Broadstairs, Kent CT10 2LE, United Kingdom

$\S$ Current address: School of Engineering \& Applied Science, Aston University, Birmingham, United Kingdom

||Current address: DSM Ahead B.V., Urmonderbaan 22, 6167 RD Geleen, The Netherlands $\perp$ Diamond Light Source Ltd., Diamond House, Harwell Science and Innovation Campus, Didcot, United Kingdom

E-mail: gregory.smith@nbi.ku.dk

\begin{abstract}
A series of ionic diblock copolymer nanoparticles was prepared in a typical nonpolar solvent ( $n$-dodecane) via polymerization-induced self-assembly (PISA). A single cationic repeat unit was incorporated into the poly(stearyl methacrylate) (PSMA) stabilizer of otherwise uncharged poly(stearyl methacrylate)-poly(benzyl methacrylate) (PSMA-PBzMA) diblock copolymers nanoparticles. By using short PSMA stabilizer
\end{abstract}


blocks, it was possible to obtain nanoparticles with the range of morphologies expected (spheres, worms, and vesicles). For nanoparticles where all stabilizer chains possessed an ionic group, higher-order morphologies were obtained at lower BzMA degrees of polymerization than corresponding uncharged particles, and the particles were electrophoretic. For nanoparticles where only a fraction of the stabilizer chains contained an ionic group, higher-order morphologies were obtained at precisely the same PBzMA degrees of polymerization, and the electrophoretic response was greater than when the shell was fully ionic. These particles with a partially ionic shell are a fascinating system, providing morphologies that can be predicted from existing knowledge of the diblock copolymer morphology yet with the highest possible electrophoretic mobility.

\section{Introduction}

Diblock copolymer nanoparticles are interesting materials that form an assortment of nanostructures in a variety of solvents (water, alcohols, and oils). ${ }^{1-3}$ Polymerization-induced selfassembly (PISA) is a versatile method for preparing such diblock copolymer nanoparticles with a wide range of possible morphologies using a variety of different polymer blocks. Aside from the typical spheres, worms, and vesicles, more exotic morphologies, such as lamellae,

framboidal vesicles, jellyfish, and yolk/shell particles, can be obtained. ${ }^{4}$ By employing a controlled radical synthesis technique, reversible addition-fragmentation chain-transfer (RAFT) polymerization, ${ }^{5}$ it is possible to synthesize polymers with well-defined blocks and low dispersities. This makes it possible to selectively and precisely insert functional monomers at specific locations into the block copolymers. An interesting example was recently reported for series of nanoparticles synthesized using two different $\mathrm{pH}$ responsive RAFT chain-transfer agents (CTAs). These CTAs possessed a single ionizable carboxylic acid group, but nevertheless, it was possible to induce a transition between different morphologies by changing pH. ${ }^{6-8}$ The concept of $\mathrm{pH}$ cannot be extended directly to nonpolar solvents, such as used here, because the solvents are not autoionizable, and to achieve anything similar to $\mathrm{pH}$-induced 
protonation requires the addition of an external ionizing agent. ${ }^{9}$

This example of changing $\mathrm{pH}$ demonstrates the challenge of trying to universalize stimuli responsiveness between different solvents. Nonpolar solvents provide a particularly challenging medium to generate particles that respond to electrostatic stimuli. It is very challenging to introduce ionic moieties into nonpolar solvents, due to their low dielectric constant. Once introduced, though, the Coulombic interactions are long-range. ${ }^{10}$ In this study, we have examined the impact that incorporating a single charged group can have on the morphology of nanoparticles in a nonaqueous, specifically nonpolar, low dielectric solvent. Nonpolar fluids are not only interesting as potential media for the production of nanoparticles. ${ }^{3}$ They are relevant media due to their use as industrial solvents for a variety of purposes. ${ }^{11}$ The production of charge in nonpolar solvent is of particular interest due the technologically advanced materials that make use of the response of these ions to applied electric fields, such as electrophoretic displays ${ }^{12,13}$ and electrorheological fluids. ${ }^{14}$

Using the PISA approach, the model system of uncharged poly(stearyl methacrylate)poly(benzyl methacrylate) (PSMA-BzMA) diblock copolymers synthesized in $n$-dodecane, ${ }^{15,16}$ which is known to produce nanoparticles with a sphere, worm, or vesicle morphologies for sufficiently low SMA degrees of polymerization, was modified from the simple case by the incorporation of a polymerizable ionic monomer. The ionic monomer used ((2(methacryloyloxy)ethyl)trimethylammonium tetrakis[3,5-bis(trifluoromethyl)phenyl]borate, $\left.[\mathrm{MOTMA}]^{+}[\mathrm{TFPhB}]^{-}\right)$is from a class of functional molecules that is known to produce electrophoretic spheres in nonpolar solvents. ${ }^{17-19}$ Guided by the extensive studies of nanoparticles synthesized by PISA in water that have shown that nonspherical morphologies can rationally be generated ${ }^{1,20}$ and that ions ${ }^{6-8,21}$ can be used to control their self-assembly, we have introduced this ionic monomer into diblock copolymers that should form nonspherical morphologies. RAFT polymerization ${ }^{5}$ provides the precision to locate the ionic monomer into the stabilizer of the PSMA-PBzMA nanoparticles, so that we can assess the impact that it has on the morphologies formed as well as on the electrokinetic properties. This is a straight- 
forward way to introduce this functional monomer selectively, but as only a single ionic unit is incorporated into the stabilizer (on average), there will be a population of uncharged stabilizer molecules as well as populations with more than one ionic unit. The full range of expected pure morphologies (spheres, worms, and vesicles) have successfully been produced using both uncharged PSMA $13-$ PBzMA diblock copolymers (Figure 1(a)) and charged shell $\left[\mathrm{P}\left(\mathrm{SMA}_{13} \text {-stat-MOTMA } 1\right)\right]^{+}\left[\mathrm{MOTMA}^{-}\right.$(Figures $1(\mathrm{~b})$ and $\left.(\mathrm{c})\right)$. By placing the ionic groups in the shell, they can interact at the surface. The effective projected area of the stabilizer chains then increases. This shifts the critical degree of polymerization (DP) for morphology formation as compared to the uncharged case. The charged shell nanoparticles are also electric field responsive, undergoing counterion condensation, ${ }^{22,23}$ a characteristic of charged species in low dielectric media. ${ }^{19,24}$ Nanoparticles were also prepared where only a fraction of the stabilizer chains were ionic, and these give morphology boundaries that are the same as the uncharged case but, surprisingly, have a greater electric field response than nanoparticles with a fully charged shell stabilizer.

The motivation for undertaking this is twofold. Nano-objects synthesized by PISA have been shown to be promising materials for industrial applications, ${ }^{25}$ but varying the DP of the core-forming block is still the primary way of controlling the morphologies that are produced in a PISA synthesis, as was the case when these type of materials were first reported. ${ }^{26}$ Charged polymer colloids in nonpolar solvents are employed in industrial technology, such as electrophoretic displays, ${ }^{12,13}$ but conventional latex polymerization has only been used to resulted in spherical nanoparticles. ${ }^{27}$ This study addresses both issues. We demonstrate a new way of influencing the morphology of nano-objects in a nonpolar solvent, using ionic stabilizer groups, and we show that it is possible to produce electric field responsive nonspherical nanoparticles in a nonpolar solvent, using PISA. 


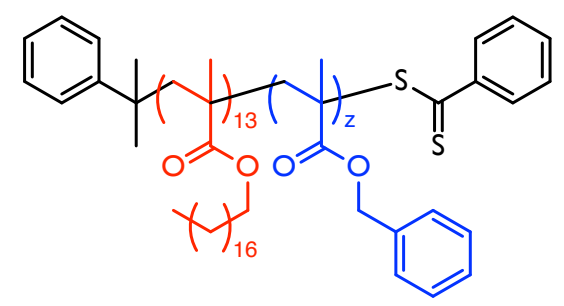

(a) Uncharged diblock copolymer

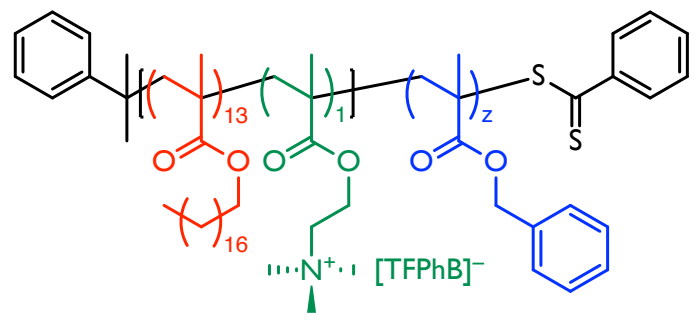

(b) Charged shell diblock copolymer

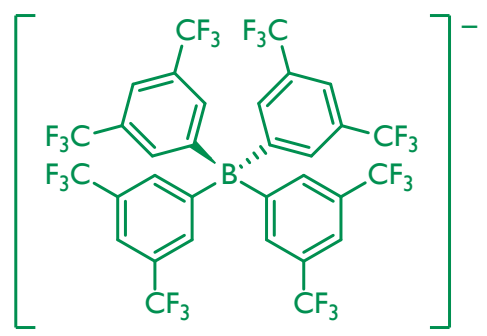

(c) Tetrakis(3,5-bis(trifluoromethyl)phenyl)borate anion ([TFPhB] $]^{-}$)

Figure 1: The structure of the diblock copolymers used to form nanoparticles. (a) Uncharged poly(stearyl methacrylate)-poly(benzyl methacrylate (PSMA-PBzMA) diblock copolymers. (b) Charged shell [poly((stearyl methacrylate)-stat-(((2(methacryloyloxy)ethyl)trimethylammonium) $)$-poly(benzyl methacrylate) $]^{+}$[tetrakis$[3,5 \text {-bis(trifluoromethyl)phenyl]borate }]^{-} \quad\left(\left[\mathrm{P}_{\left(\mathrm{SMA}_{13} \text {-stat-MOTMA }\right.}\right)-\mathrm{PBzMA}^{+}[\mathrm{TFPhB}]^{-}\right)$ diblock copolymers. (c) The $[\mathrm{TFPhB}]^{-}$anion that is the counterion for the charged shell diblock copolymers. 


\section{Materials and Methods}

\section{Polymer synthesis}

Stearyl methacrylate (SMA, technical grade) and benzyl methacrylate (BzMA, 96\%) monomers were purchased from Sigma-Aldrich (UK). BzMA monomer was passed through a basic alumina column to remove inhibitor prior to use. The ionic monomer (2-(methacryloyloxy)ethyl)trimethylammonium tetrakis[3,5-bis(trifluoromethyl)phenyl]borate $\left([\mathrm{MOTMA}]^{+}[\mathrm{TFPhB}]^{-}\right)$was produced as previously reported. ${ }^{19} 2,2^{\prime}$-Azobisisobutyronitrile initiator was purchased from Molekula (UK), and tert-butyl peroxy-2-ethylhexanoate (Trigonox 21S, T21s) initiator was a gift from AkzoNobel (The Netherlands). Cumyl dithiobenzoate (CDB, 99\%) was purchased from Sigma-Aldrich and used as supplied. Toluene solvent (HPLC grade) was purchased from Fisher (UK) and used as supplied. $n$-Dodecane ( $\geq 99 \%)$ solvent used for synthesis and to prepare dispersions was obtained from Sigma-Aldrich (UK) and was stored over molecular sieves prior to use.

\section{PSMA macromolecular chain-transfer agents (macro-CTAs)}

An uncharged poly(stearyl methacrylate) (PSMA) macro-CTA with a degree of polymerization (DP) of 13 was synthesized and purified using the same method as applied to higher DP analogs. ${ }^{19}$ An ionic statistical copolymer of SMA and one $[\mathrm{MOTMA}]^{+}[\mathrm{TFPhB}]^{-}$repeat unit (on average) was synthesized using the same protocol, but the purification was modified due to the increased solubility of the polymer in short chain alcohols. There is only an average of one ionic unit per chain, and so there will be populations of uncharged polymer as well populations with more than one. It has not been possible to determine this distribution. It was precipitated from ice cold methanol to remove unreacted $[\mathrm{MOTMA}]^{+}[\mathrm{TFPhB}]^{-}$, and then precipitated in ice cold ethanol to remove unreacted SMA. Characterization of the macro-CTA polymers are given in Supporting Information (Tables S1 and S2). 


\section{PSMA-PBzMA diblock copolymer nanoparticles}

Diblock copolymers were synthesized by dissolving the appropriate macro-CTA, BzMA monomer, and T21S initiator (added as a 10 wt. \% solution in $n$-dodecane; macro-CTA/T21S molar ratio $=3)$ ) in $n$-dodecane to give a copolymer concentration of $12.5 \mathrm{wt}$. \%. The reaction was allowed to proceed for a minimum of 18 hours, and conversions of $>90 \%$ were obtained. Analytical chemistry and purity are provided in the Supporting Information (Tables S3-S5).

\section{Small-angle X-ray scattering (SAXS)}

SAXS measurements were performed on the instrument I22 at Diamond Light Source (Rutherford Appleton Laboratory, UK). The instrument configuration provided a $Q$-range of $0.0017-$

$0.2 \AA^{-1}$, where $Q$ is the modulus of the momentum transfer vector, calculated from $\theta$ (half the scattering angle) and $\lambda$ (the wavelength of the radiation).

$$
Q=\frac{4 \pi \sin \theta}{\lambda}
$$

The scattering intensity was collected on a 2D Pilatus 2M pixel detector. Scattering from samples and solvents were collected in $1.8 \mathrm{~mm}$ inner diameter polycarbonate capillaries, and two-dimensional scattering patterns were radially integrated, normalized, and solvent subtracted using instrument-specific software, Dawn, ${ }^{28}$ using established data reduction pipelines. ${ }^{29}$ Details of data analysis and models are given in the Supporting Information.

\section{Dynamic and electrophoretic light scattering}

Dynamic light scattering (DLS) was used to determine the solvodynamic size of the nanoparticles. Electrophoretic light scattering, specifically phase-analysis light scattering (PALS) was used to determine the electrophoretic mobility of the nanoparticles. Both measurements were performed on a Malvern Zetasizer Nano ZS instrument. 
DLS measurements were performed at $25{ }^{\circ} \mathrm{C}$ on dispersions diluted to $0.1 \mathrm{wt}$. $\%$ with $n$-dodecane. Three measurements of approximately ten runs of $10 \mathrm{~s}$ duration were averaged. From cumulants analysis of the correlogram (in the Malvern Zetasizer software), the solvodynamic $Z$-average particle diameter $\left(d_{Z}=2 r_{Z}\right)$ was determined from the diffusion coefficient $(D)$ via the Stokes-Einstein equation, ${ }^{30,31}$ where $k_{B} T$ is the thermal energy and $\eta$ is the solvent viscosity: $D=\left(k_{B} T\right) /(6 \pi \eta r)$. To compare $Z$-average diameters properly requires that the dispersion have a monomodal distribution and that particles are nearly-spherical in shape. ${ }^{32}$ These conditions will be met for spherically symmetric objects (spheres and vesicles) but not for elongated objects (worms), so in instances where this is the case, the value of $d_{Z}$ is illustrative only. A deconvolution algorithm (in the Malvern Zetasizer software) was used to determine the intensity-weighted size distribution. ${ }^{32}$

PALS ${ }^{33}$ measurements were performed at $25{ }^{\circ} \mathrm{C}$ using a universal dip cell electrode ${ }^{34}$ on dispersions diluted to a volume fraction $\phi$ of $10^{-4}$. The volume fraction was kept constant as the electrophoretic mobility of charged particles in salt-free media in the high-charge limit does not depend on the charge density but does depend on the volume fraction. ${ }^{35-40}$ The applied field strength was $2 \times 10^{4} \mathrm{~V} \mathrm{~m}^{-1}$. Six runs of 50 measurements were averaged to give a value of the electrophoretic mobility $\mu$.

\section{Results and Discussion}

To understand how the incorporation of ionic moieties into the shell of PSMA-PBzMA nanoparticles impacts their morphology and their properties, we consider two aspects in turn. Morphology maps showing how the nanoparticles change as a function of the DP of the structure-directing PBzMA block were produced. These are the essential way to identify the rational evolution of morphologies. ${ }^{4}$ The magnitudes of the electrophoretic mobilities as a function of the nanoparticle sizes were obtained, and these are expected to vary in a characteristic way for particles undergoing counterion condensation. ${ }^{19,24}$ Both of these will 
reveal if the incorporation of ions into the shell of PSMA-PBzMA nanoparticles impacts their morphology and properties.

\section{Diblock copolymer morphology}

A diblock copolymer nanoparticle morphology map (the diblock copolymer morphology as a function of core DP) has been produced to show what block compositions are required to obtain certain morphologies. Figure 2 shows morphology maps for uncharged $\mathrm{PSMA}_{13^{-}}$ $\mathrm{PBzMA}_{z}$ and charged shell $\left[\mathrm{P}\left(\mathrm{SMA}_{13} \text {-stat-MOTMA } 1\right)-\mathrm{PBzMA}_{z}\right]^{+}[\mathrm{TFPhB}]^{-}$diblock copolymer nanoparticles synthesized at 12.5 wt. \% in $n$-dodecane. Morphologies were assigned by analysis of transmission electron microscopy images, dynamic light scattering diameters and size distributions, and small-angle X-ray scattering data.DLS intensity distributions and representative TEM micrographs for both of these cases (charged shell and uncharged) are shown in Figures 3 and 4 . The uncharged case is very similar to previously reported for a PSMA $_{13}$ macro-CTA stabilizer by Derry et al. ${ }^{15}$ The slight differences arise from batch-to-batch variations in synthesizing the PSMA macromolecular chain-transfer agent (macro-CTA). Pure sphere, worm, and vesicle morphologies can be produced with using both macro-CTAs. Between these pure morphologies are regions where mixtures of morphologies are obtained. For the charged shell nanoparticles, there is a region at large PBzMA DPs where PISA fails to produce well-defined nano-objects. We refer to these large and ill-defined nanostructures as "compound micelles", a term used in the literature previously to describe instances where short stabilizers can no longer maintain colloidal stability and the growing micelles fuse together. ${ }^{41,42}$ The DLS intensity distribution and a representative TEM image of these compound micelles are shown in Figure 5.

The progression of morphologies for the charged shell $\left[\mathrm{P}\left(\mathrm{SMA}_{13^{-}} \text {stat-MOTMA } 1\right)\right]^{+}[\mathrm{TFPhB}]^{-}$ stabilizer is exactly the same as formed from the uncharged $\mathrm{PSMA}_{13}$ one. However, the formation of higher-order morphologies is induced at a lower core DP. The morphology boundary is shifted by $\sim 10-20$ BzMA units compared to the uncharged case. This sug- 


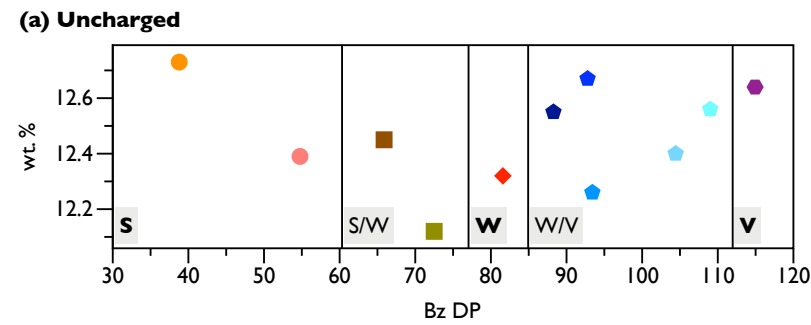

(b) Charged shell

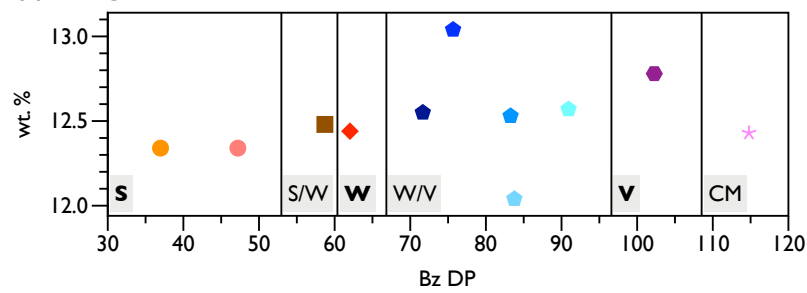

Figure 2: Morphology maps for (a) uncharged and (b) charged shell PSMA-PBzMA diblock copolymers nanoparticles synthesized at $\sim 12.5$ wt. $\% \%$ in $n$-dodecane. The $x$-axis is the degree of polymerization (DP) of the PBzMA core, and the $y$-axis is the precise diblock copolymer concentration in wt. \%. Nanoparticles with pure spherical, worm-like, and vesicular morphologies are all obtained. The charged shell particles produce higher-order morphologies at lower PBzMA DPs than the corresponding uncharged ones. The regions in the morphology map are labelled with a letter denoting the morphology, with single letters representing pure morphologies and combinations of letters representing mixed morphologies. (S (spheres), W (worms), V (vesicles), and CM (compound micelles).) 


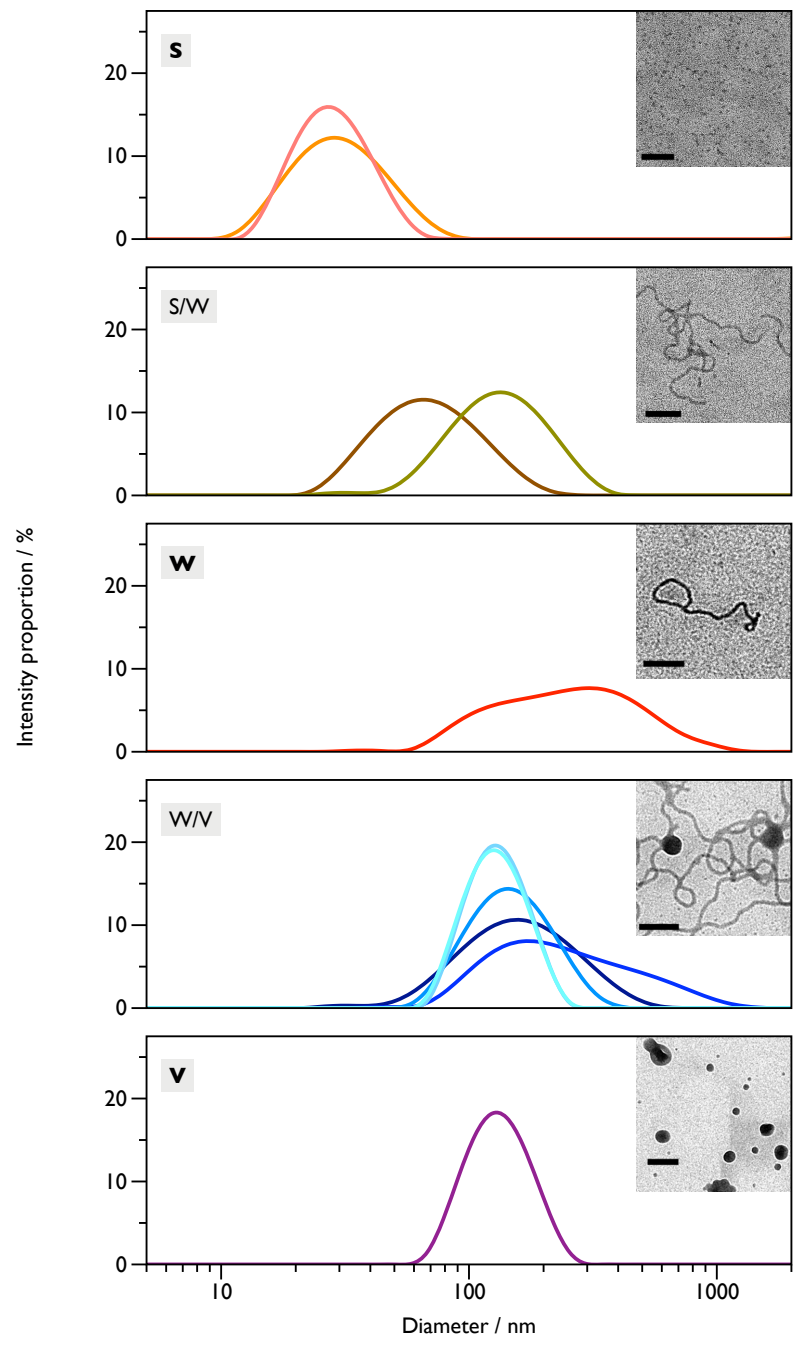

Figure 3: Intensity-weighted particle size distributions from DLS for uncharged $\mathrm{PSMA}_{13}{ }^{-}$ PBzMA nano-objects synthesized at $\sim 12.5$ wt. \% in $n$-dodecane. The single letters represent pure morphologies and combinations of letters represent mixed morphologies. (S (spheres), $\mathrm{W}$ (worms), and V (vesicles).) The colors of the curves correlate to the data points in Figure 2. The insets are representative TEM micrographs of each morphology, each with a scale bar of $200 \mathrm{~nm}$. 


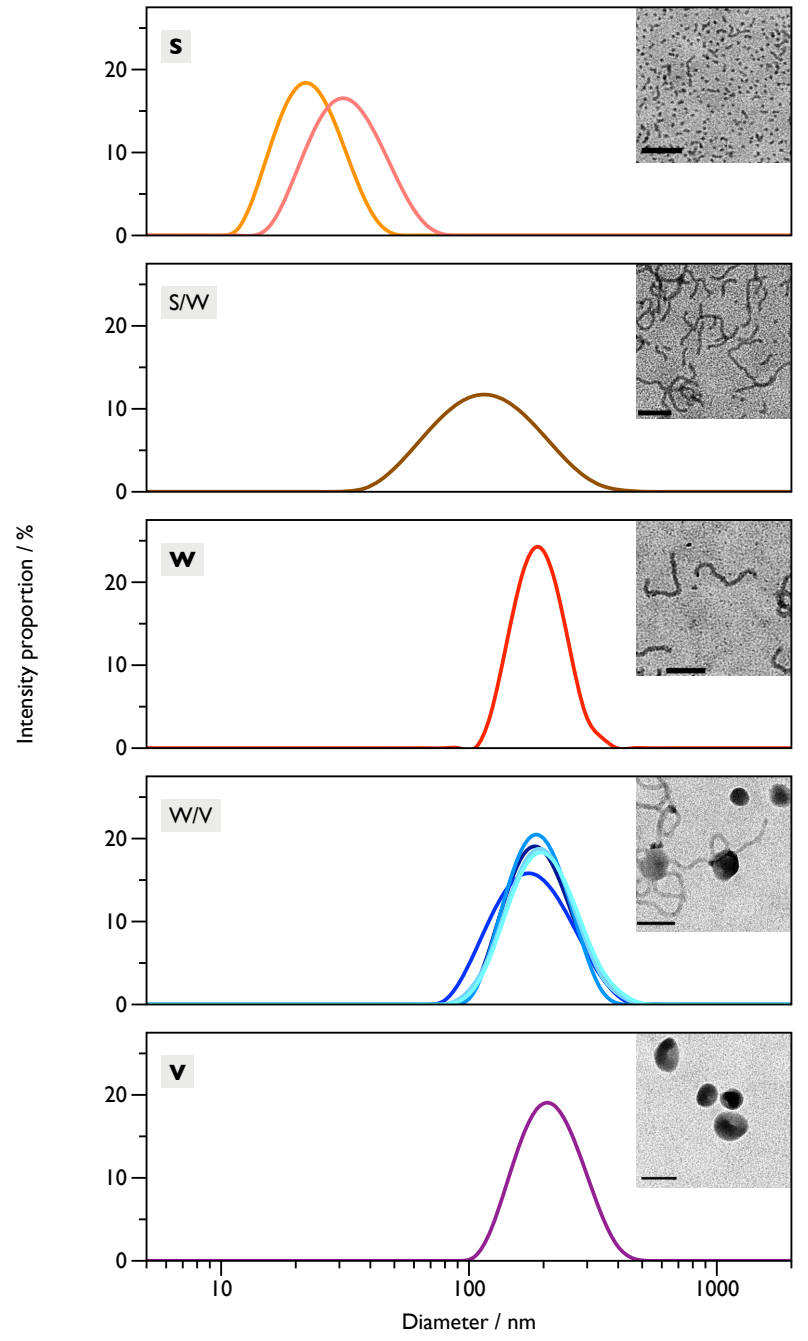

Figure 4: Intensity-weighted particle size distributions from DLS for charged shell $\left[\mathrm{P}\left(\mathrm{SMA}_{13^{-}}\right.\right.$ stat-MOTMA 1$)-\mathrm{PBzMA}^{+}[\mathrm{TFPhB}]^{-}$nano-objects synthesized at $\sim 12.5 \mathrm{wt}$. $\%$ in $n$ dodecane. The single letters represent pure morphologies and combinations of letters represent mixed morphologies. (S (spheres), W (worms), and $\mathrm{V}$ (vesicles).) The colors of the curves correlate to the data points in Figure 2. The insets are representative TEM micrographs of each morphology, each with a scale bar of $200 \mathrm{~nm}$.

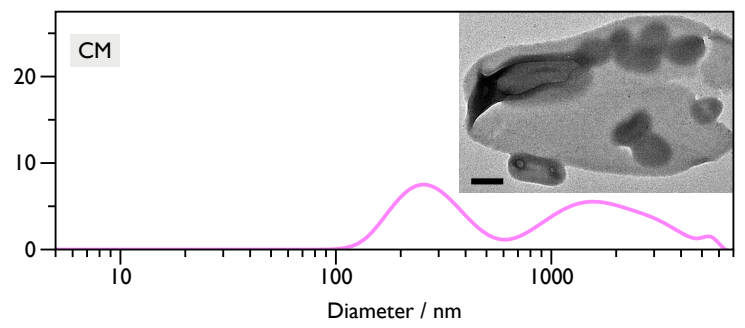

Figure 5: Intensity-weighted particle size distribution from DLS for charged shell $\left[\mathrm{P}\left(\mathrm{SMA}_{13^{-}}\right.\right.$ stat-MOTMA 1$\left.)-\mathrm{PBzMA}_{115}\right]^{+}[\mathrm{TFPhB}]^{-}$compound micelles $(\mathrm{CM})$ synthesized at $\sim 12.5 \mathrm{wt}$. $\%$ in $n$-dodecane. The inset is an illustrative TEM micrograph, with a scale bar of $200 \mathrm{~nm}$. 
gests that the $\left[\mathrm{P}\left(\mathrm{SMA}_{13} \text {-stat-MOTMA }\right)\right]^{+}[\mathrm{TFPhB}]^{-}$polymer is a poorer steric stabilizer for nanoparticles, because higher-order morphologies form at a lower DP. Alternatively, one can consider packing arguments to come to the same conclusion. Coulombic repulsion between charged groups on the surface of a nanoparticle will increase their effective surface area $\left(a_{0}\right)$. This will decrease the value of the packing parameter $\left(P \propto a_{0}^{-1}\right),{ }^{43}$ promoting the transition between spheres-to-worms and worms-to-vesicles at lower core volume $v$, which is directly proportional to the degree of polymerization of the structure-directing core block.

As pure morphologies can be produced, small-angle X-ray scattering (SAXS) was employed to determine quantitative structural parameters to enable a comparison between nanoparticles stabilized by uncharged and charged PSMA chains. The SAXS data, along with fits to appropriate models (discussed in the Supporting Information), are shown in Figure 6. The comparable uncharged and charged shell morphologies can be fit with nearly identical geometric dimensions. This is only for parameters that can be well resolved in the accessible $Q$ range, which is related to the real-space length scale $(d)$ through Bragg's Law $\left(Q=(2 \pi) / d^{44}\right)$. For the instrument used, this corresponds to feature sizes between 370 and $3 \mathrm{~nm}(3700$ and $30 \AA)$. Of the fit parameters that can be resolved within the $Q$-range of the measurements, the sphere radius, worm radius, and vesicle wall thickness all deviate less than $\pm 1 \mathrm{~nm}$ between the uncharged and charged shell cases. Therefore, it appears that the ionic monomer impacts the critical DP for forming nanoparticles of a certain morphology, but it does not impact the structure of the nanoparticles that are formed.

From the fit dimensions, it is possible to calculate the nanoparticle volume $\left(v_{n}\right)$ and surface area $\left(S A_{n}\right)$. Then, using the volume of a single PBzMA chain in the core $\left(v_{c}\right)$, it is possible to calculate either the number of chains per surface area $\left(n_{s}\right)$ or the aggregation number of chains in the core $\left(n_{\text {agg }}\right)$.

$$
n_{s}=\frac{S A_{n}}{n_{a g g}}=\frac{S A_{n}}{v_{n} / v_{c}}
$$



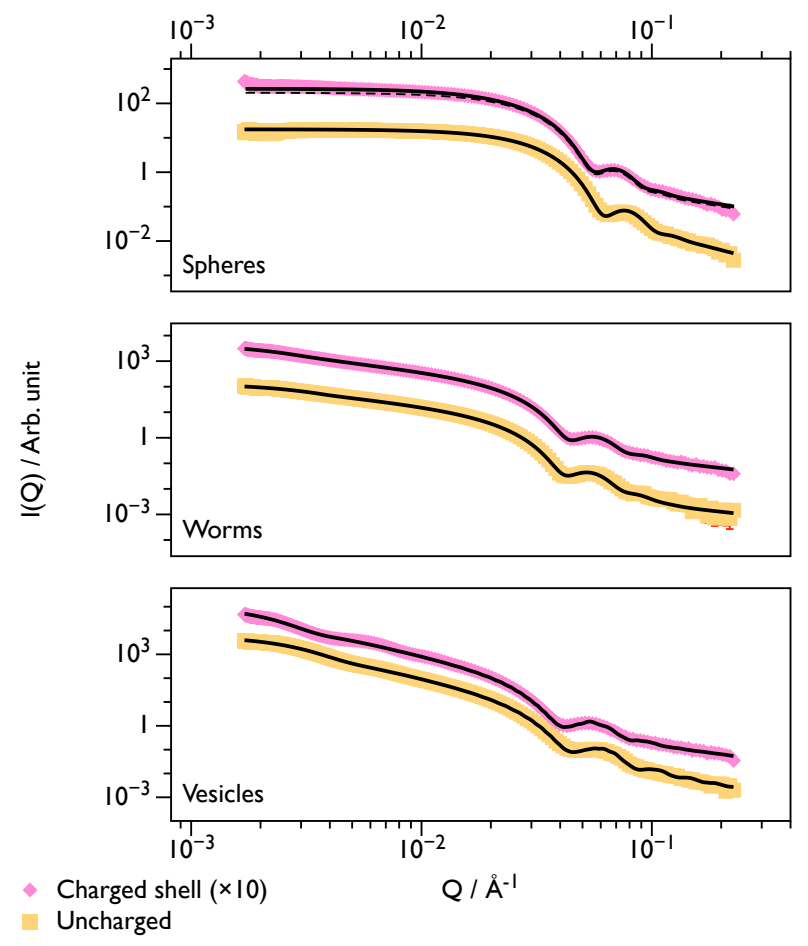

Figure 6: Small-angle X-ray scattering data for uncharged and charged shell particles. Welldefined nanoparticles can be obtained in both cases, which is clear from the scattering curves and the quality of the fits to the data. (Note, there is a small population of sphere dimers present in the charged shell dispersion, and the fit to a sphere model only is shown by the dashed line.) 
The determined values are given in the Supporting Information (Tables S9, S11, and S13). The value of $n_{\text {agg }}$ for the uncharged nanoparticles is less than for the shell charged nanoparticles. This is because the morphologies occur at higher PBzMA DPs and, consequently, higher $v_{c}$ in the nonionic case. Therefore, fewer PBzMA chains are required to fill the core volume of the uncharged nanoparticles.

We have established that it is possible to make pure morphologies using both of these diblock copolymer systems. Referring back to Figure 2, what occurs in the case where fractional amounts of charged and uncharged macro-CTA are used to prepare nanoparticles? For larger PSMA stabilizers where only spherical nanoparticles are formed, the size of the particles changes, but no higher-order morphologies are formed. ${ }^{19}$ Will the strong interactions between the charged chains always dominate? A series of nanoparticles were prepared with $10 \%$ charged shell macro-CTA to study this.

A morphology map of these nanoparticles is shown in Figure 7(a). DLS intensity distributions and representative TEM micrographs are shown in Figure 8. The pure morphologies in the morphology maps for the uncharged and charged shell diblock copolymers are shown in Figure 7(b) as well for reference. It is immediately apparent that the pure morphology boundaries occur at essentially the same critical PBzMA DPs as for the uncharged case (Figure 7(b)). Therefore, if the fraction of ion-containing PSMA macro-CTA is significantly reduced, the evolution of morphologies of the nanoparticles is exactly the same as if stabilized by the uncharged stabilizer.

\section{Electrokinetics of ionic nanoparticles}

The morphology of these nanoparticles stabilized with a fractionally charged shell PSMA shell evolves the same as in the uncharged case. This raises the question: how does the electrophoresis of the nanoparticles compare to the fully charged shell or uncharged case? Are they unresponsive like the uncharged nanoparticles, or are they electrophoretic like the

charged shell nanoparticles? Normalized electrophoretic mobilities of the fully charged shell 

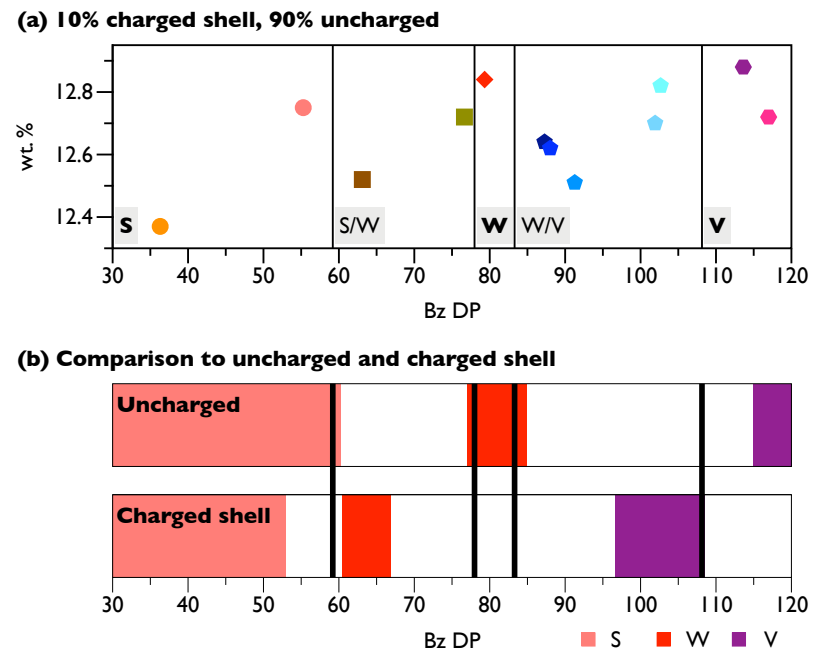

Figure 7: Morphology map for (a) partially charged shell (10\%) particles compared to (b) the fully uncharged and fully charged shell cases. The axes are the same as in Figure 2. Spherical, worm-like, and vesicular morphologies are also all obtained for this mix of stabilizers. The solid vertical lines in (b) are projections of the pure morphology boundaries onto the uncharged and charged shell morphology maps. The regions in the morphology map are labelled with a letter denoting the morphology, with single letters represent pure morphologies and combinations of letters represent mixed morphologies. ( $\mathrm{S}$ (spheres), W (worms), and V (vesicles).) In terms of the morphologies that they form, the partly charged polymers appear to be similar to the uncharged case. 

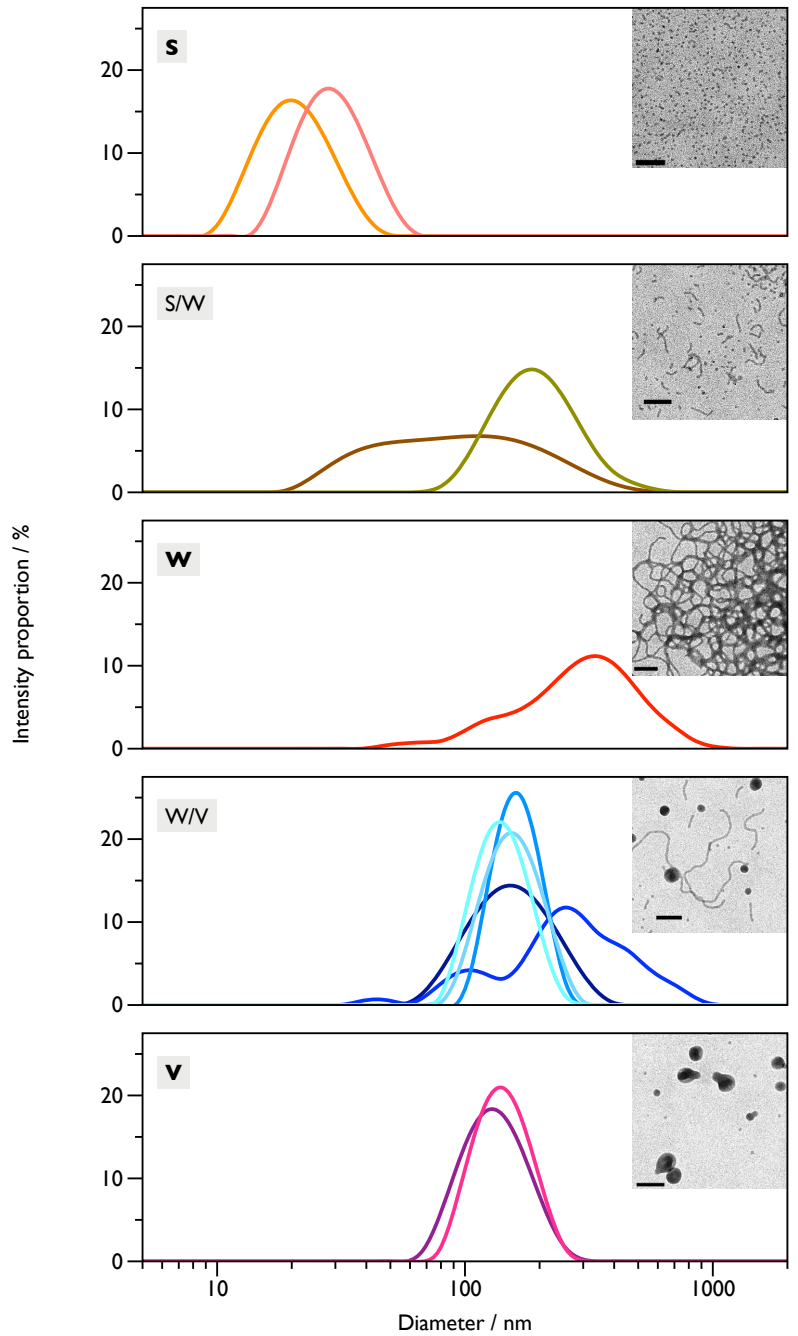

Figure 8: Intensity-weighted particle size distributions from DLS for partly charged shell (0.9) $\left(\mathrm{PSMA}_{13}-\mathrm{PBzMA}\right)-(0.1)\left(\left[\mathrm{P}\left(\mathrm{SMA}_{13} \text {-stat-MOTMA } 1\right)-\mathrm{PBzMA}\right]^{+}[\mathrm{TFPhB}]^{-}\right) \quad$ nanoobjects synthesized at $\sim 12.5$ wt. $\%$ in $n$-dodecane. The single letters represent pure morphologies and combinations of letters represent mixed morphologies. ( $\mathrm{S}$ (spheres), W (worms), and V (vesicles).) The colors of the curves correlate to the data points in Figure 7. The insets are representative TEM micrographs of each morphology, each with a scale bar of $200 \mathrm{~nm}$. 
and the partially charged shell nanoparticles (10\% ionic stabilizer) are shown in Figure 9 for all particles as a function of the $Z$-average diameter measured by DLS $\left(d_{Z}\right)$. These diameters are not a geometric diameter for the specific morphology, rather they are a sphere-equivalent solvodynamic diameter. Not all of the size distributions have a monomodal distribution or are nearly-spherical in shape (Figures 3,4,5, and 8), which are the requirements to compare $d_{Z}$ between different particles. ${ }^{32}$ This means that the DLS-determined sizes cannot necessarily be compared. However, the electrokinetics of charged particles, particularly shell charged particles, depends on the solvodynamics around the particle, ${ }^{45}$ and so the solvodynamic diameter, as determined by DLS, is appropriate for this purpose. The error bars shown for $d_{Z}$ are not experimental uncertainties but instead are the standard deviations determined from the DLS measurements. If the distribution of decay rates of the autocorrelation functions is Gaussian, the DLS-determined "polydispersity" index (PdI) can be related to the distribution of particle sizes. (Strictly this relationship is for the distribution of decay rates, which are inversely related to the particles sizes. $\left.{ }^{46}\right)$ The standard deviation $(\sigma)$ can be calculated from the DLS-determined PdI and the diameter $\left(d_{Z}\right)$, using Equation $3 .{ }^{47}$

$$
\sigma=\sqrt{\mathrm{PdI}} \cdot d_{Z}
$$

The electrophoretic mobilities are normalized by the term $\mu_{0}$, equal to $e /\left(6 \pi \eta \lambda_{B}\right) .\left(\lambda_{B}=\right.$ $e^{2} /\left(4 \pi \epsilon_{0} \epsilon_{r} k_{B} T\right)$ is the Bjerrum length, ${ }^{48} e$ is the elementary charge, $\eta$ is the fluid viscosity, $\epsilon_{0}$ is the vacuum permittivity, $\epsilon_{r}$ is the relative permittivity, and $k_{B} T$ is the thermal energy.)

In a previous report in the literature of spheres undergoing counterion condensation in a salt-free nonpolar solvent, $\mu$ was found to increase linearly for small particles and plateau for large particles for particles at the same volume fraction $\phi .{ }^{24}$ That is indeed what is observed in the case of both ion-containing PSMA-PBzMA nanoparticles. In this case, however, the diameters are sphere-equivalent diameters, not actual spherical diameters. What is particularly remarkable is that the magnitude of the plateau of the electrophoretic mobility is 
greater for the partially charged shell nanoparticles than the fully charged shell nanoparticles. Therefore, reducing the interactions between charged chains on the surfaces increases their responsiveness to an electric field. This was previously observed for electrophoretic PSMAPBzMA spheres,${ }^{19}$ but it would not have been possible to predict that this would also be observed with nonspherical nanoparticles with only a single ionic monomer unit the PSMA stabilizer. The ability to explain this observation is beyond current electrokinetic theories, and a new theory for charged shell spheres in salt-free media will be required.
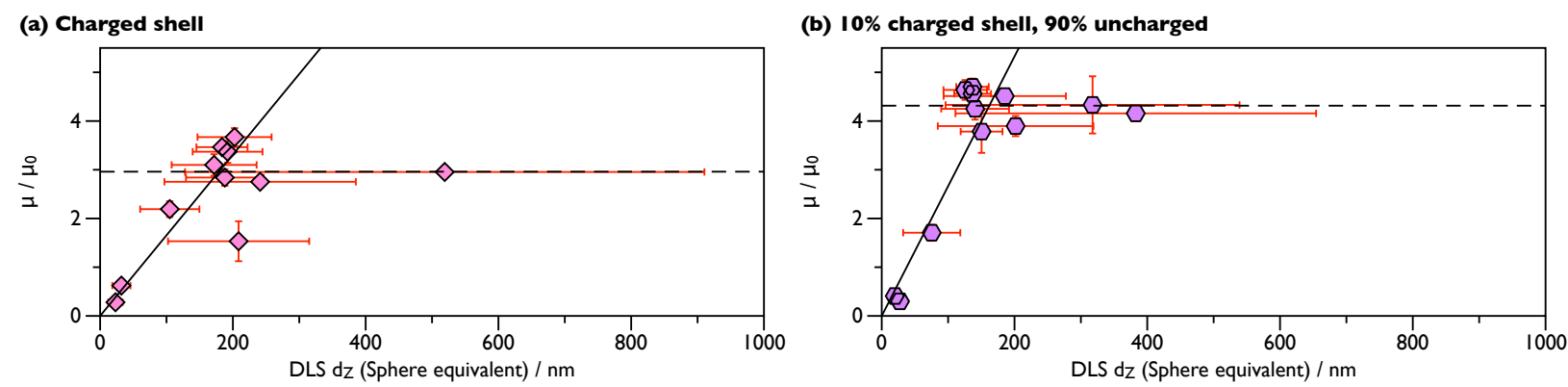

Figure 9: Normalized electrophoretic mobility $\left(\mu / \mu_{0}\right)$ for (a) charged shell and (b) partially charged shell (10\%) nanoparticles as a function of particle size (DLS $\left.d_{Z}\right)$. The error bars are not experimental uncertainties but represent the standard deviation $(\sigma)$ calculated from the DLS-determined polydispersity index, as explained in the text. As expected for objects undergoing counterion condensation, the mobility increases linearly when the objects are small, and it plateaus when they are large. The partly charged shell particles have a higher magnitude mobility, despite the fact that they contain fewer ionic units.

\section{Conclusions}

Nonspherical nanoparticles have been produced in a nonpolar solvent that respond to an applied electric field. This was achieved by the incorporation of a polymerizable ionic monomer into the macro-CTA stabilizer. Due to the low relative permittivity of such solvents, the presence of an ionic group in the particle shell has a dramatic effect on the morphology, shifting the onset of nonspherical morphologies (worm-like micelles and vesicles) to lower DPs than for the uncharged case. Diluting the charged interactions at the surface using a fraction of ionic macro-CTA chains results in a very interesting system of nanoparticles, 
where the morphologies can be predicted from the uncharged case but the electrophoretic mobility is greater than for the fully charged shell case. This system, in particular, is very promising for enabling the rational synthesis of highly electrophoretic nanoparticles. The extensive knowledge of how to synthesize nonspherical nanoparticles by PISA in nonpolar solvents using poly(lauryl methacrylate), ${ }^{26,49,50}$ poly(dimethylsiloxane), ${ }^{51-53}$ poly(lauryl acrylate), ${ }^{54,55}$ poly(stearyl methacrylate), ${ }^{15,16,56-60}$ poly(2-(methacryloyloxy)ethyl oleate) ${ }^{61}$ and poly(3-[tris(trimethylsiloxy)silyl]propyl methacrylate) ${ }^{62}$ stabilizers can be directly applied to enable the production of electrophoretic nanoparticles. Such systems would offer the promise of generating interesting charged shell functional nanomaterials. Depending on the morphology, these could provide a variety of charge functionalized nanoparticles. Further studies to explore the properties of these and related nanoparticles in nonpolar fluids are highly warranted.

\section{Acknowledgement}

GNS acknowledges Professor S. P. Armes (University of Sheffield, UK) for hosting him while this work was performed and funding from the ERC (a five-year Advanced Investigator grant (PISA 320372)) and EPSRC (EP/J007846) while working in his group. Diamond Light Source is acknowledged for awarding time on I22. The UK Science and Technologies Facility Council (STFC) is acknowledged for funding travel costs associated with visiting the Diamond Light Source.

\section{Supporting Information Available}

Characterization of the diblock copolymers and diblock copolymer nanoparticles. Details of SAXS models and analysis. 


\section{References}

(1) Warren, N. J.; Armes, S. P. Polymerization-Induced Self-Assembly of Block Copolymer Nano-objects via RAFT Aqueous Dispersion Polymerization. J. Am. Chem. Soc. 2014, 136, 10174-10185, DOI: 10.1021/ja502843f.

(2) Lowe, A. B. RAFT alcoholic dispersion polymerization with polymerization-induced self-assembly. Polymer 2016, 106, 161-181.

(3) Derry, M. J.; Fielding, L. A.; Armes, S. P. Polymerization-induced self-assembly of block copolymer nanoparticles via RAFT non-aqueous dispersion polymerization. Prog. Polym. Sci. 2016, 52, 1-18, DOI: 10.1016/j.progpolymsci.2015.10.002.

(4) Canning, S. L.; Smith, G. N.; Armes, S. P. A Critical Appraisal of RAFT-Mediated Polymerization-Induced Self-Assembly. Macromolecules 2016, 49, 1985-2001, DOI: 10.1021/acs.macromol.5b02602.

(5) Moad, G.; Rizzardo, E.; Thang, S. H. Living Radical Polymerization by the RAFT Process. Aust. J. Chem. 2005, 58, 379-410, DOI: 10.1071/CH05072.

(6) Lovett, J. R.; Warren, N. J.; Armes, S. P.; Smallridge, M. J.; Cracknell, R. B. Order-Order Morphological Transitions for Dual Stimulus Responsive Diblock Copolymer Vesicles. Macromolecules 2016, 49, 1016-1025, DOI: 10.1021/acs.macromol.5b02470.

(7) Penfold, N. J. W.; Lovett, J. R.; Warren, N. J.; Verstraete, P.; Smets, J.; Armes, S. P. pH-Responsive non-ionic diblock copolymers: protonation of a morpholine end-group induces an order-order transition. Polym. Chem. 2016, 7, 79-88, DOI: 10.1039/C5PY01510C.

(8) Penfold, N. J. W.; Lovett, J. R.; Verstraete, P.; Smets, J.; Armes, S. P. Stimulusresponsive non-ionic diblock copolymers: protonation of a tertiary amine end-group 
induces vesicle-to-worm or vesicle-to-sphere transitions. Polym. Chem. 2017, 8, 272282, DOI: $10.1039 /$ C6PY01076H.

(9) Smith, G. N. Proton transfer in nonpolar solvents: an approach to generate electrolytes in aprotic media. Phys. Chem. Chem. Phys. 2018, 20, 18919-18923, DOI: 10.1039/C8CP02349B.

(10) Morrison, I. D. Electrical charges in nonaqueous media. Colloids Surf. A: Physicochem. Eng. Aspects 1993, 71, 1-37, DOI: 10.1016/0927-7757 (93)80026-B.

(11) Cheremisinoff, N. P. Industrial Solvents Handbook, 2nd ed.; Marcel Dekker: New York, 2003.

(12) Ota, I.; Ohnishi, J.; Yoshiyama, M. Electrophoretic image display (EPID) panel. Proc. IEEE 1973, 61, 832-836, DOI: 10.1109/PROC.1973.9173.

(13) Comiskey, B.; Albert, J. D.; Yoshizawa, H.; Jacobson, J. An electrophoretic ink for allprinted reflective electronic displays. Nature 1998, 394, 253-255, DOI: 10. 1038/28349.

(14) Hao, T. Electrorheological Fluids. Adv. Mater. 2001, 13, 1847-1857, DOI: 10.1002/1521-4095(200112) 13:24<1847: :AID-ADMA1847>3. 0.CO;2-A.

(15) Derry, M. J.; Fielding, L. A.; Warren, N. J.; Mable, C. J.; Smith, A. J.; Mykhaylyk, O. O.; Armes, S. P. In situ small-angle X-ray scattering studies of sterically-stabilized diblock copolymer nanoparticles formed during polymerizationinduced self-assembly in non-polar media. Chem. Sci. 2016, 7, 5078-5090, DOI: 10.1039/C6SC01243D.

(16) Derry, M. J.; Mykhaylyk, O. O.; Armes, S. P. A Vesicle-to-Worm Transition Provides a New High-Temperature Oil Thickening Mechanism. Angew. Chem. Int. Ed. 2017, 56, 1746-1750, DOI: 10.1002/anie.201609365. 
(17) Sánchez, R.; Bartlett, P. Synthesis of charged particles in an ultra-low dielectric solvent. Soft Matter 2011, 7, 887-890, DOI: 10.1039/COSM01454K.

(18) Greinert, N.; Uerdingen, M.; Beylage, L.; Ignatyev, N.; Wilson, J. H.; Goulding, M. J.; Kemp, R.; Smith, A. N.; Bartlett, P.; Barthen, P.; Frank, W.; Garcia, R. S. Particles for electrophoretic displays. Patent WO 2012/072218 A1, 2012.

(19) Smith, G. N.; Mears, L. L. E.; Rogers, S. E.; Armes, S. P. Synthesis and electrokinetics of cationic spherical nanoparticles in salt-free non-polar media. Chem. Sci. 2018, 9, 922-934, DOI: 10.1039/C7SC03334F.

(20) Blanazs, A.; Ryan, A. J.; Armes, S. P. Predictive Phase Diagrams for RAFT Aqueous Dispersion Polymerization: Effect of Block Copolymer Composition, Molecular Weight, and Copolymer Concentration. Macromolecules 2012, 45, 5099-5107, DOI: $10.1021 / \operatorname{ma301059r.}$

(21) Williams, M.; Penfold, N. J. W.; Lovett, J. R.; Warren, N. J.; Douglas, C. W. I.; Doroshenko, N.; Verstraete, P.; Smets, J.; Armes, S. P. Bespoke cationic nano-objects via RAFT aqueous dispersion polymerisation. Polym. Chem. 2016, 7, 3864-3873, DOI: 10.1039/C6PY00696E.

(22) Manning, G. S. Limiting Laws and Counterion Condensation in Polyelectrolyte Solutions I. Colligative Properties. J. Chem. Phys. 1969, 51, 924-933, DOI: $10.1063 / 1.1672157$.

(23) Oosawa, F. Polyelectrolytes; Marcel Dekker: New York, 1971.

(24) Gillespie, D. A. J.; Hallett, J. E.; Elujoba, O.; Che Hamzah, A. F.; Richardson, R. M.; Bartlett, P. Counterion condensation on spheres in the salt-free limit. Soft Matter 2014, 10, 566-577, DOI: 10.1039/C3SM52563E. 
(25) Derry, M. J.; Smith, T.; O'Hora, P. S.; Armes, S. P. Block Copolymer Nanoparticles Prepared via Polymerization-Induced Self-Assembly Provide Excellent Boundary Lubrication Performance for Next-Generation Ultralow-Viscosity Automotive Engine Oils. ACS Appl. Mater. Interfaces 2019, 11, 33364-33369, DOI: 10.1021/acsami. $9 b 12472$.

(26) Fielding, L. A.; Derry, M. J.; Ladmiral, V.; Rosselgong, J.; Rodrigues, A. M.; Ratcliffe, L. P. D.; Sugihara, S.; Armes, S. P. RAFT dispersion polymerization in non-polar solvents: facile production of block copolymer spheres, worms and vesicles in $n$-alkanes. Chem. Sci. 2013, 4, 2081-2087, DOI: 10.1039/C3SC50305D.

(27) Smith, G. N.; Hallett, J. E.; Eastoe, J. Celebrating Soft Matter's 10th Anniversary: Influencing the charge of poly(methyl methacrylate) latexes in nonpolar solvents. Soft Matter 2015, 11, 8029-8041, DOI: 10.1039/C5SM01190F.

(28) Filik, J.; Ashton, A. W.; Chang, P. C. Y.; Chater, P. A.; Day, S. J.; Drakopoulos, M.; Gerring, M. W.; Hart, M. L.; Magdysyuk, O. V.; Michalik, S.; Smith, A.; Tang, C. C.; Terrill, N. J.; Wharmby, M. T.; Wilhelm, H. Processing two-dimensional X-ray diffraction and small-angle scattering data in DAWN 2. J. Appl. Cryst. 2017, 50, 959-966, DOI: $10.1107 / \mathrm{S} 1600576717004708$.

(29) Pauw, B. R.; Smith, A. J.; Snow, T.; Terrill, N. J.; Thünemann, A. F. The modular small-angle X-ray scattering data correction sequence. J. Appl. Cryst. 2017, 50, 18001811, DOI: $10.1107 / \mathrm{S} 1600576717015096$.

(30) Einstein, A. Eine neue Bestimmung der Moleküldimensionen. Ann. Phys. 1906, 324, 289-306, DOI: 10.1002/andp.19063240204.

(31) Einstein, A. Berichtigung zu meiner Arbeit: „Eine neue Bestimmung der Moleküldimensione". Ann. Phys. 1911, 339, 591-592, DOI: 10.1002/andp.19113390313.

(32) Malvern Instruments, Dynamic Light Scattering: Common Terms Defined. White Paper, 2011. 
(33) Miller, J. F.; Schätzel, K.; Vincent, B. The determination of very small electrophoretic mobilities in polar and nonpolar colloidal dispersions using phase analysis light scattering. J. Colloid Interface Sci. 1991, 143, 532-554, DOI: https://doi .org/10.1016/0021-9797(91)90286-H.

(34) Tucker, I.; Corbett, J.; Fatkin, J.; Jack, R.; Kaszuba, M.; MacCreath, B.; McNeilWatson, F. Laser Doppler Electrophoresis applied to colloids and surfaces. Curr. Opin. Colloid Interface Sci. 2015, 20, 215-226, DOI: 10.1016/j.cocis.2015.07.001.

(35) Ohshima, H. Surface Charge Density/Surface Potential Relationship for a Spherical Colloidal Particle in a Salt-Free Medium. J. Colloid Interface Sci. 2002, 247, 18-23, DOI: $10.1006 /$ jcis. 2001.8105.

(36) Ohshima, H. Electrophoretic Mobility of a Spherical Colloidal Particle in a Salt-Free Medium. J. Colloid Interface Sci. 2002, 248, 499-503, DOI: 10.1006/jcis .2002.8232.

(37) Ohshima, H. Numerical calculation of the electrophoretic mobility of a spherical particle in a salt-free medium. J. Colloid Interface Sci. 2003, 262, 294-297, DOI: 10.1016/S0021-9797(03)00190-5.

(38) Ohshima, H. Potential distribution around a polyelectrolyte-coated spherical particle in a salt-free medium. J. Colloid Interface Sci. 2003, 268, 429-434, DOI: $10.1016 / j \cdot j$ cis. 2003.08 .020 .

(39) Ohshima, H. Electrophoretic mobility of a soft particle in a salt-free medium. J. Colloid Interface Sci. 2004, 269, 255-258, DOI: 10.1016/S0021-9797(03)00600-3.

(40) Ohshima, H. Erratum to "Electrophoretic mobility of a soft particle in a salt-free medium": [J. Colloid Interface Sci. 269 (2004) 255-258]. J. Colloid Interface Sci. 2004, 272, 503, DOI: 10.1016/j.jcis.2003.12.063. 
(41) Zhang, L.; Eisenberg, A. Formation of crew-cut aggregates of various morphologies from amphiphilic block copolymers in solution. Polym. Adv. Technol. 1998, 9, 677-699, DOI: 10.1002/(SICI) 1099-1581(1998100)9:10/11<677: :AID-PAT845>3. 0.CO;2-\\#.

(42) Warren, N. J.; Mykhaylyk, O. O.; Ryan, A. J.; Williams, M.; Doussineau, T.; Dugourd, P.; Antoine, R.; Portale, G.; Armes, S. P. Testing the Vesicular Morphology to Destruction: Birth and Death of Diblock Copolymer Vesicles Prepared via Polymerization-Induced Self-Assembly. J. Am. Chem. Soc. 2015, 137, 1929-1937, DOI: 10.1021/ja511423m.

(43) Israelachvili, J. The science and applications of emulsions - an overview. Colloids Surf. A: Physicochem. Eng. Aspects 1994, 91, 1-8, DOI: 10.1016/0927-7757(94)02743-9.

(44) Grillo, I. In Soft Matter Characterization; Borsali, R., Pecora, R., Eds.; Springer Netherlands, 2008; pp 723-782, DOI: 10.1007/978-1-4020-4465-6\_13.

(45) Delgado, A. V.; González-Caballero, F.; Hunter, R. J.; Koopal, L. K.; Lyklema, J. Measurement and Interpretation of Electrokinetic Phenomena (IUPAC Technical Report). Pure Appl. Chem. 2005, 77, 1753-1805, DOI: 10.1351/pac200577101753.

(46) King, S.; Kienzle, P. Polydispersity \& Orientational Distributions (SasView 4.2.2 documentation). http://www.sasview.org/docs/user/sasgui/perspectives/fitting/ $\mathrm{pd} /$ polydispersity.html.

(47) Hirschle, P.; Preiß, T.; Auras, F.; Pick, A.; Völkner, J.; Valdepérez, D.; Witte, G.; Parak, W. J.; Rädler, J. O.; Wuttke, S. Exploration of MOF nanoparticle sizes using various physical characterization methods - is what you measure what you get? CrystEngComm 2016, 18, 4359-4368, DOI: 10.1039/C6CE00198J.

(48) Bjerrum, N. Untersuchungen Über Ionenassoziation. I. Der Einfluss der Ionenassoziation auf die Aktivität der Ionen bei Mittleren Assoziationsgraden. Kgl. Danske Vidensk. Selsk., Math.-fys. Medd. 1926, 7, 1-48. 
(49) Fielding, L. A.; Lane, J. A.; Derry, M. J.; Mykhaylyk, O. O.; Armes, S. P. Thermoresponsive Diblock Copolymer Worm Gels in Non-polar Solvents. J. Am. Chem. Soc. 2014, 136, 5790-5798, DOI: 10.1021/ja501756h.

(50) Derry, M. J.; Fielding, L. A.; Armes, S. P. Industrially-relevant polymerization-induced self-assembly formulations in non-polar solvents: RAFT dispersion polymerization of benzyl methacrylate. Polym. Chem. 2015, 6, 3054-3062, DOI: 10.1039/C5PY00157A.

(51) Lopez-Oliva, A. P.; Warren, N. J.; Rajkumar, A.; Mykhaylyk, O. O.; Derry, M. J.; Doncom, K. E. B.; Rymaruk, M. J.; Armes, S. P. Polydimethylsiloxane-Based Diblock Copolymer Nano-objects Prepared in Nonpolar Media via RAFT-Mediated Polymerization-Induced Self-Assembly. Macromolecules 2015, 48, 3547-3555, DOI: 10.1021/acs.macromol.5b00576.

(52) Rymaruk, M. J.; O'Brien, C. T.; Brown, S. L.; Williams, C. N.; Armes, S. P. Effect of Core Cross-linking on the Physical Properties of Poly(dimethylsiloxane)-Based Diblock Copolymer Worms Prepared in Silicone Oil. Macromolecules 2019, 52, 6849-6860, DOI: 10.1021/acs.macromol.9b01488.

(53) Rymaruk, M. J.; Hunter, S. J.; O'Brien, C. T.; Brown, S. L.; Williams, C. N.; Armes, S. P. RAFT Dispersion Polymerization in Silicone Oil. Macromolecules 2019, 52, 2822-2832, DOI: 10.1021/acs.macromol.9b00129.

(54) Ratcliffe, L. P. D.; McKenzie, B. E.; Bouëdec, G. M. D. L.; Williams, C. N.; Brown, S. L.; Armes, S. P. Polymerization-Induced Self-Assembly of All-Acrylic Diblock Copolymers via RAFT Dispersion Polymerization in Alkanes. Macromolecules 2015, 48, 8594-8607, DOI: 10.1021/acs.macromol.5b02119.

(55) Canning, S. L.; Cunningham, V. J.; Ratcliffe, L. P. D.; Armes, S. P. Phenyl acrylate is a versatile monomer for the synthesis of acrylic diblock copolymer nano-objects 
via polymerization-induced self-assembly. Polym. Chem. 2017, 8, 4811-4821, DOI: 10.1039/C7PY01161J.

(56) Pei, Y.; Thurairajah, L.; Sugita, O. R.; Lowe, A. B. RAFT Dispersion Polymerization in Nonpolar Media: Polymerization of 3-Phenylpropyl Methacrylate in nTetradecane with Poly(stearyl methacrylate) Homopolymers as Macro Chain Transfer Agents. Macromolecules 2015, 48, 236-244, DOI: 10.1021/ma502230h.

(57) Pei, Y.; Noy, J.-M.; Roth, P. J.; Lowe, A. B. Soft Matter Nanoparticles with Reactive Coronal Pentafluorophenyl Methacrylate Residues via Non-Polar RAFT Dispersion Polymerization and Polymerization-Induced Self-Assembly. J. Polym. Sci. A: Polym. Chem. 2015, 53, 2326-2335, DOI: 10.1002/pola.27696.

(58) Pei, Y.; Sugita, O. R.; Thurairajah, L.; Lowe, A. B. Synthesis of poly(stearyl methacrylate- $b$-3-phenylpropyl methacrylate) nanoparticles in $n$-octane and associated thermoreversible polymorphism. $R S C A d v$. 2015, 5, 17636-17646, DOI: 10. 1039/C5RA00274E.

(59) Cunningham, V. J.; Armes, S. P.; Musa, O. M. Synthesis, characterisation and Pickering emulsifier performance of poly(stearyl methacrylate)-poly( $N$-2-(methacryloyloxy)ethyl pyrrolidone) diblock copolymer nano-objects via RAFT dispersion polymerisation in n-dodecane. Polym. Chem. 2016, 7, 1882-1891, DOI: 10.1039/C6PY00138F.

(60) Smith, G. N.; Canning, S. L.; Derry, M. J.; Mykhaylyk, O. O.; Norman, S. E.; Armes, S. P. Influence of an ionic comonomer on polymerization-induced selfassembly of diblock copolymers in non-polar media. Polym. Chem. 2020, DOI: 10.1039/DOPY00101E.

(61) Maiti, B.; Bauri, K.; Nandi, M.; De, P. Surface functionalized nano-objects from oleic acid-derived stabilizer via non-polar RAFT dispersion polymerization. J. Polym. Sci. A: Polym. Chem. 2017, 55, 263-273, DOI: 10.1002/pola. 28373. 
(62) Rymaruk, M. J.; O’Brien, C. T.; Brown, S. L.; Williams, C. N.; Armes, S. P. RAFT Dispersion Polymerization of Benzyl Methacrylate in Silicone Oil Using a Silicone-Based Methacrylic Stabilizer Provides Convenient Access to Spheres, Worms, and Vesicles. Macromolecules 2020, 53, 1785-1794, DOI: 10.1021/acs.macromol.9b02697. 


\section{Graphical TOC Entry}

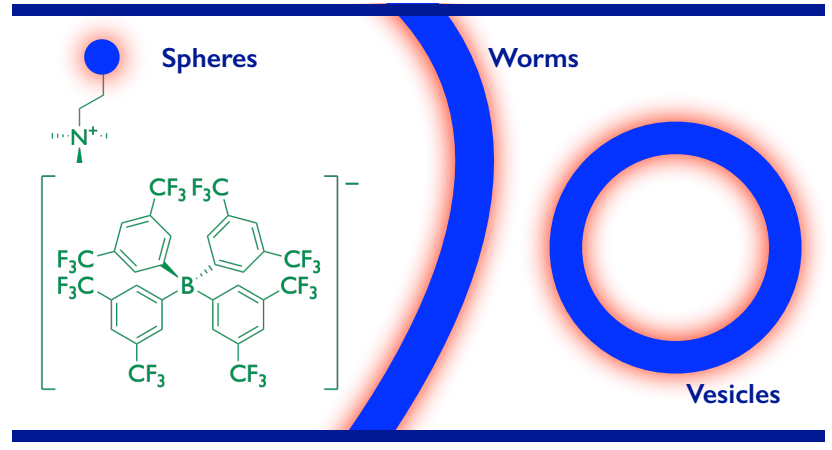

